\title{
FUSION FREE REPRESENTATIONS OF FINITE GROUPS
}

MARC J. LIPMAN

I. Introduction. Let $G$ be a finite group. Let $Q$ denote the field of rational numbers. If $\sigma \in G,\langle\sigma\rangle$ is the group generated by $\sigma,|\sigma|$ is the order of $\langle\sigma\rangle$, and if $S$ and $S^{\prime} \subset G, S \sim S^{\prime}$ means $S$ and $S^{\prime}$ are conjugate subsets of $G$. It is well known that the following definitions are equivalent:

Definition. $G$ is a $Q$-group if every complex character of $G$ is $Q$-valued.

Definition. $G$ has cyclic conjugacy if for every $\sigma$ and $\tau \in G,\langle\sigma\rangle \sim\langle\tau\rangle$ iff $\sigma \sim \tau$. Equivalently, $G$ has cyclic conjugacy if for every $\sigma$ and $\tau \in G$ such that $\langle\sigma\rangle=\langle\tau\rangle$, then $\sigma \sim \tau$.

This paper presents two other criteria for $Q$-groups, one in terms of permutation representations, one in terms of rational representations. The essential concept is the "fusion free representation".

Definition. Let $f: G \rightarrow H$ be a homomorphism of groups. $f$ is fusion free if for every $\sigma$ and $\tau \in G: \sigma \sim \tau$ in $G$ iff $f(\sigma) \sim f(\tau)$ in $H$. This is denoted by $G \subset H$. If $f$ is fusion free then $f$ is $1-1$. Thus we consider $G$ to be a subgroup of $H$, justifying the notation. A fusion free representation of $G$ is any representation of $G$ consisting of a fusion free homomorphism.

The main results of this paper are:

THEOREM 2.5. Let $G$ be a finite group. $G$ is a $Q$-group iff for some $n \geqq 1$, $G \subset S_{n}$.

COROLlary 4.3. Let $G$ be a finite group. $G$ is a $Q$-group iff for some $n \geqq 1$, $G \subset \operatorname{GL}(n, Q)$.

Let $\sigma \in S_{n}$. The "type" of $\sigma$ is an $n$-tuple $\left(c_{1}, \ldots, c_{n}\right)$, where $c_{i}$ is the number of cycles of length $i$ in $\sigma$. For $\sigma$ and $\tau \in S_{n}, \sigma \sim \tau$ iff $\sigma$ and $\tau$ have the same type. Further, if $\langle\sigma\rangle=\langle\tau\rangle$ then $\sigma$ and $\tau$ have the same cycle structure, that is, $\sigma \sim \tau$. Thus for all $n \geqq 1, S_{n}$ is a $Q$-group.

Let $k$ be a field with $\operatorname{char}(k) \chi n !$. ( $n$ ! is the order of $S_{n}$.) Choose an ordered basis for an $n$-dimensional vector space over $k$. Define the natural mapping nat: $S_{n} \rightarrow \operatorname{GL}(n, k)$ by assigning to each $\sigma \in S_{n}$ the permutation matrix in $\operatorname{GL}(n, k)$ associated to $\sigma$. In Section III the following theorem is proved: 
THEOREM 3.4. Let $k$ be a field with $\operatorname{char}(k) \nmid n$ !. Then, using the natural embedding, $S_{n} \subset \mathrm{GL}(n, k)$.

\section{Fusion Free Permutation Representations.}

Proposition 2.1. If $G \subset S_{n}$, then $G$ is a $Q$-group.

Proof. Suppose $\langle\sigma\rangle=\langle\tau\rangle$ for $\sigma$ and $\tau \in G$. Then $\sigma \sim \tau$ in $S_{n}$ since $S_{n}$ is a $Q$-group. $G \subset S_{n}$ implies $\sigma \sim \tau$ in $G$.

The goal for the remainder of this section is to prove the converse of Proposition 2.1. Let $G$ be a $Q$-group. Let $\sigma_{1}, \ldots, \sigma_{s}$ be a full set of representatives for the conjugacy classes of $G$, ordered so that $\left|\sigma_{i}\right| \leqq\left|\sigma_{i+1}\right|$ for $i=1, \ldots, s-1$.

Consider the characters $\alpha_{i}=1{ }_{\left\langle\sigma_{i}\right\rangle}^{G}$ for $i=1, \ldots, s . \alpha_{i}$ is the character of $G$ of the permutation representation $\left(G, G /\left\langle\sigma_{i}\right\rangle\right)$. That is, $\alpha_{i}$ is the character of the representation of $G$ acting by left multiplication on the left cosets of $\left\langle\sigma_{i}\right\rangle$ in $G$.

Proposition 2.2. Let $G$ be a $Q$-group. With the above notation, if $j>i$ then $\alpha_{i}\left(\sigma_{j}\right)=0$.

PROOF. $\alpha_{i}\left(\sigma_{j}\right) \neq 0$ iff for some $\tau \in G, \sigma_{j} \tau\left\langle\sigma_{i}\right\rangle=\tau\left\langle\sigma_{i}\right\rangle$ iff $\sigma_{j} \sim \sigma_{i}^{k}$ for some integer $k$.

If $j>i,\left|\sigma_{j}\right| \geqq\left|\sigma_{i}\right|$ and $\left\langle\sigma_{j}\right\rangle \nsim\left\langle\sigma_{i}\right\rangle$ since $G$ is a $Q$-group. Thus $\sigma_{j} \nsim \sigma_{i}^{k}$ for any $k$. Thus $\alpha_{i}\left(\sigma_{j}\right)=0$.

Note that $\alpha_{i}\left(\sigma_{i}\right)=\left[N_{G}\left(\left\langle\sigma_{i}\right\rangle\right):\left\langle\sigma_{i}\right\rangle\right]=$ the index of $\left\langle\sigma_{i}\right\rangle$ in its normalizer in $G \neq 0$.

THEOREM 2.3. Let $G$ be a $Q$-group. Use the above notation. There exists a proper permutation character $\chi$ of $G$ so that if $i \neq j$ then $\chi\left(\sigma_{i}\right) \neq \chi\left(\sigma_{j}\right)$.

Proof. Let $\chi=\sum_{l=1}^{s} a_{l} \alpha_{l}$ with the $a_{l}$ chosen as follows: Let $a_{s}=1$. For $j=s-1, \ldots, 1$ let $a_{j}=1+\sum_{k=j+1}^{s} a_{k} \alpha_{k}\left(\sigma_{j+1}\right)$. Then for all $i>j$, $\sum_{k=j}^{s} a_{k} \alpha_{k}\left(\sigma_{j}\right)>\sum_{k=i}^{s} a_{k} \alpha_{k}\left(\sigma_{i}\right)$. By Proposition $2.2 \chi\left(\sigma_{i}\right)=\sum_{k=i}^{s} a_{k} \alpha_{k}\left(\sigma_{i}\right)$, so that if $i>j \chi\left(\sigma_{j}\right)>\chi\left(\sigma_{i}\right)$.

Theorem 2.4. Let $G$ be a $Q$-group. For some $n \geqq 1, G \subset S_{n}$.

Proof. Choose $\chi$ as in Theorem 2.3. Let $n=\chi(1)$. Let $X: G \rightarrow S_{n}$ be the permutation representation afforded by $\chi$. Then via $X, G \subset S_{n}$.

Proposition 2.1 and Theorem 2.4 immediately give

THEOREM 2.5. Let $G$ be a finite group. $G$ is a $Q$-group iff for some $n>1$, $G \subset S_{n}$.

III. Fusion Free Representation of $S_{n}$. Let $k$ be an algebraically 
closed field with char $(k) \chi n$ ! Consider the natural embedding nat: $S_{n} \rightarrow$ $\mathrm{GL}(n, k)$ described in Section I. Let $\sigma \in S_{n}$ and suppose $\sigma$ has type $\left(c_{1}, \ldots, c_{n}\right)$. Considering $\sigma$ as a permutation matrix in $\operatorname{GL}(n, k)$ define $w_{j}$ to be the multiplicity of any primitive $j^{\text {th }}$ root of unity as an eigenvalue of $\sigma$.

Lemma 3.1: $w_{j}$ is well defined. For all $j=1, \ldots, n w_{j}=c_{j}+c_{2 j}+c_{3 j}+$ $\ldots=\sum_{i ; j \mid i} c_{i}$.

Proof. $\sigma$ is similar to a permutation matrix that is the direct sum of matrices of cycles. Thus the characteristic polynomial of $\sigma$ is the product of the characteristic polynomials of the cycles of $\sigma$. A cycle of length $i$ has characteristic polynomial $X^{i}-1$. If $\zeta$ is a primitive $j^{\text {th }}$ root of unity, then $\zeta$ is a root of $X^{i}-1$ iff $j \mid i$. Further, if $j \mid i$ then $\zeta$ is a root of $X^{i}-1$ of multiplicity exactly 1 . Hence $w_{j}=$ the number of cycles of length a multiple of $j$, giving the result.

Lemma 3.2. For all $j=1, \ldots, n, c_{j}=\sum_{i} \mu(i) w_{i j}$, where $\mu$ is the classical Möbius function.

Proof. Möbius inversion on the partially ordered set of the integers with the dual division ordering: $i \leqq j$ iff $j \mid i$. (See [1], p. 83.)

THEOREM 3.3. Using the natural embedding, $S_{n} \mp \mathrm{GL}(n, k)$.

Proof. Choose $\sigma$ and $\tau \in S_{n}$. If $\sigma \sim \tau$ in $\operatorname{GL}(n, k)$ then $\sigma$ and $\tau$ have the same eigenvalue structure. By Lemma 3.2 this eigenvalue structure determines a unique type. Thus $\sigma \sim \tau$ in $S_{n}$.

THEOREM 3.4. Let $k$ be any field with $\operatorname{char}(k) \nmid n$ ! Using the natural embedding, $S_{n} \subset \mathrm{GL}(n, k)$.

Proof. Let $K$ be the algebraic closure of $k$. Then $S_{n} \subset \mathrm{GL}(n, K)$ and $S_{n} \subseteq \mathrm{GL}(n, k) \subseteq \mathrm{GL}(n, K)$. If $\sigma$ and $\tau \in S_{n}$ and $\sigma \sim \tau$ in $\operatorname{GL}(n, k)$, then $\sigma \sim \tau$ in $\operatorname{GL}(n, K)$. Thus $\sigma \sim \tau$ in $S_{n}$ so $S_{n} \subset \operatorname{GL}(n, k)$.

Corollary 3.5. Let $G$ be a $Q$-group. Let $k$ be a field with $\operatorname{char}(k)=0$. For some $n \geqq 1, G \subset \mathrm{GL}(n, k)$.

Proof. For some $n \geqq 1, G \subset S_{n}$. Since $\operatorname{char}(k)=0, S_{n} \subset \mathrm{GL}(n, k)$. Thus $G \subset \operatorname{GL}(n, k)$.

iV. Fusion Free Rational Representations. Denote the $s^{\text {th }}$ cyclotomic polynomial over $Q$ by $\varphi_{s}(X)$.

Lemma 4.1. Let $M$ and $N \in \mathrm{GL}(n, Q)$ be matrices of finite order $r$. Suppose $\langle M\rangle=\langle N\rangle$. Then $M \sim N$.

Proof. $N=M^{a}$ for some a with $(a, r)=1 . M$ satisfies $X^{r}-1=$ $\Pi_{s \mid r} \varphi_{s}(X)$. Thus the minimal polynomial of $M, m(X)=\Pi_{s \mid r}\left(\varphi_{s}(X)\right)^{e_{s}}$, 
where $e_{s}=0$ or 1 for all $s$, since each $\varphi_{s}(X)$ is irreducible and all are distinct. The characteristic polynomial of $M, c(X)$, can be written as the product of the $\varphi_{s}(X)$ appearing in $m(X)$. That is:

$$
c(X)=\prod_{(s \mid r)}\left(\varphi_{s}(X)\right)^{f_{s}}, \quad f_{s} \neq 0 \text { iff } e_{s}=1 .
$$

If $\zeta$ is an eigenvalue of $M$ of multiplicity $f$, then $\zeta^{a}$ is an eigenvalue of $M^{a}=N$ of multiplicity at least $f$. Since $\zeta$ is a root of $\varphi_{s}(X)$ for some $s \mid r$ and $(a, r)=1$, it follows that $\zeta^{a}$ is a root of the same $\varphi_{s}(X)$. That is, if $d(X)$ is the characteristic polynomial of $N$,

$$
d(X)=\prod_{(s \mid r)}\left(\varphi_{s}(X)\right)^{g_{s}}, g_{s} \geqq f_{s} .
$$

But degree $(c(X))=n=$ degree $(d(X))$, so $c(X)=d(X)$.

Because $m(X)$ is the product of distinct irreducibles, the rational canonical form of $M$ is completely determined by the number of blocks due to each irreducible factor of $m(X)$. That is, the numbers $f_{s}, s \mid r$, completely determine the class of $M$ in $\operatorname{GL}(n, k)$. Since $N$ also satisfies $X^{r}-1$, etc., $c(X)=d(X)$ gives $M \sim N$.

THEOREM 4.2. Let $G$ be a finite group. If $G \subset \mathrm{GL}(n, Q)$, then $G$ is a $Q$ group.

Proof. For $\sigma$ and $\tau \in G$, if $\langle\sigma\rangle=\langle\tau\rangle$ then by Lemma $4.1 \sigma \sim \tau$ in $\operatorname{GL}(n, Q)$. Then $G \subset \operatorname{GL}(n, Q)$ gives $\sigma \sim \tau$ in $G$.

COROLLARY 4.3. Let $G$ be a finite group. $G$ is a $Q$-group iff for some $n \geqq 1$, $G \subset \operatorname{GL}(n, Q)$.

Proof. In Corollary 3.5 let $k=Q$. Then Corollary 3.5 and Theorem 4.2 give the result.

\section{REFERENCE}

1. Berge, C. Principles of Combinatories, Academic Press, New York, (1971).

INDIANA University-Purdue University, Fort WAyne INDIANa 